\title{
Ultrafast laser direct hard-mask writing for high efficiency c-Si texture designs
}

\author{
Kitty Kumar ${ }^{1}$, Kenneth K.C. Lee ${ }^{2}$, Jun Nogami ${ }^{1}$, Peter R. Herman ${ }^{2}$, and Nazir P. Kherani ${ }^{1,2, a}$ \\ 1 Department of Materials Science and Engineering, 184 College Street, Toronto, Ontario, M5S 3E4, Canada \\ 2 Department of Electrical and Computer Engineering, 10 King's College Rd. Toronto, Ontario, M5S 3G4, Canada
}

Received: 5 October 2012 / Received in final form: 31 January 2013 / Accepted: 21 February 2013

Published online: 22 March 2013

(C) Kumar et al., published by EDP Sciences, 2013

\begin{abstract}
This study reports a high-resolution hard-mask laser writing technique to facilitate the selective etching of crystalline silicon (c-Si) into an inverted-pyramidal texture with feature size and periodicity on the order of the wavelength which, thus, provides for both anti-reflection and effective light-trapping of infrared and visible light. The process also enables engineered positional placement of the invertedpyramid thereby providing another parameter for optimal design of an optically efficient pattern. The proposed technique, a non-cleanroom process, is scalable for large area micro-fabrication of high-efficiency thin c-Si photovoltaics. Optical wave simulations suggest the fabricated textured surface with $1.3 \mu \mathrm{m}$ inverted-pyramids and a single anti-reflective coating increases the relative energy conversion efficiency by $11 \%$ compared to the PERL-cell texture with $9 \mu \mathrm{m}$ inverted pyramids on a $400 \mu \mathrm{m}$ thick wafer. This efficiency gain is anticipated to improve further for thinner wafers due to enhanced diffractive light trapping effects.
\end{abstract}

\section{Introduction}

A major trend driving the development of low-cost high-efficiency c-Si based photovoltaics is a reduction in material cost through the use of thinner wafers $[1,2]$. However, thin Si wafers do not readily lend themselves to highefficiency photovoltaic devices owing to large penetration depth of infrared wavelengths. Therefore, along with surface reflection reduction, an effective light-trapping scheme is imperative for the use of thinner wafers in photovoltaic devices.

In present commercial solar cells, $160-200 \mu \mathrm{m}$ thick c$\mathrm{Si}$ wafers are chemically etched in hot potassium hydroxide $(\mathrm{KOH})$ solution to form random pyramids of few to ten microns in size on the wafer surface [3] that reduces the surface reflection by promoting multiple bounces of the incident light. However, such texture with large-scale pyramids is not appropriate for thin wafers due to the high Si consumption during etching and little to no optical diffraction for efficient light-trapping. In contrast, optical modeling has shown reduced surface reflection and strong diffraction for effective light-trapping in thin wafers only when grating textures are reduced to feature size comparable with the wavelength [4]. Although various textures consisting of rods [5,6], cones [6], inverted-pyramids [7], holes (honey-comb structure) [8], grooves [9], etc. have

\footnotetext{
${ }^{a}$ e-mail: kherani@ecf.utoronto.ca
}

been applied in different lattice configurations and with different pitches to decrease reflectivity. Inverted-pyramid texture and honey-comb texture are the most preferred in practice due to the simple, cost-effective wet chemical processes that are widely available for $\mathrm{Si}$ wafers in industry [10]. Further, assessment by Zhao et al. [11] favours an inverted-pyramidal texture over a honeycomb pattern of holes. In addition, the exposed (111) planes in the inverted-pyramidal texture can be readily passivated, a critical requirement for efficient collection of photogenerated carriers. Photolithography has produced inverted-pyramidal texture with $9 \mu \mathrm{m}$ feature size that demonstrates record energy conversion efficiency in solar cells [12], while further improvements are anticipated from nano-imprint and colloidal lithography [7] that pushes the inverted-pyramid size to sub-micron scale for thin wafers. These methods have been researched extensively and have yet to be adopted for mass production.

In this paper, we present an alternative approach of patterning silicon with inverted pyramids using a noncleanroom laser direct hard-mask writing technique. The process avails femtosecond laser interaction for blistering (Fig. 1a) and catapulting (Fig. 1b) of thin-film dielectric coatings on c-Si (100) to form a high-resolution hard-mask on c-Si which in turn facilitates chemical etching of $\mathrm{Si}$ into inverted-pyramidal structure (Fig. 1c). The technique enables inverted-pyramidal texturing of $\mathrm{Si}$ with flexible pattern designs, thus exploiting the low reflection, high 
diffractive light-trapping capability and passivation benefits of such texture. While femtosecond lasers have been used to texturize c-Si with chemical assistance [13], the produced 'black' silicon has significant structural damage that prevents passivation and decreases carrier lifetime. Alternatively, femtosecond lasers have been applied to $\mathrm{SiO}_{x}$ thin films on c-Si to form micro-blisters $[13,14]$ and induce catapulting which we extend to $\mathrm{SiN}_{x}$ and further exploit here to form a high resolution hard mask for $\mathrm{KOH}$ etching.

\section{Optimization of the texturing process}

Various thicknesses of $\mathrm{SiO}_{x}$ and $\mathrm{SiN}_{x}$ films were grown to serve as hard masks (100 nm to $300 \mathrm{~nm}$ for $\mathrm{SiO}_{x} ; 20 \mathrm{~nm}$ to $266 \mathrm{~nm}$ for $\mathrm{SiN}_{x}$ ) for alkaline etching. The $\mathrm{SiN}_{x}$ layer was grown by plasma enhanced chemical vapor deposition (PECVD) on single-side polished $p$-type (100) c-Si wafers of $400 \mu \mathrm{m}$ thickness in a PlasmaLab 100 PECVD system (Oxford Instruments) at $300{ }^{\circ} \mathrm{C}$ and $650 \mathrm{mT}$ chamber pressure using a gas mixture of $5 \%$ silane in nitrogen (400 sccm), ammonia (20 sccm) and pure nitrogen $(600 \mathrm{sccm})$. The deposition was carried out at the rate of $14 \mathrm{~nm} /$ minute by using alternating combinations of high frequency $(13.56 \mathrm{MHz})$ plasma for $13 \mathrm{~s}$ and low frequency (100 KHz) plasma for $7 \mathrm{~s}$ successively. The RF power was set to $50 \mathrm{~W}$ and $40 \mathrm{~W}$ for high and low frequencies, respectively. For $\mathrm{SiO}_{x}$, the PECVD procedure was modified to $1000 \mathrm{mT}$ chamber pressure and a gas mixture of $5 \%$ silane in nitrogen $(170 \mathrm{sccm})$ and nitrous oxide $(710 \mathrm{sccm})$. The deposition was carried out at the rate of $55 \mathrm{~nm} /$ minute by using $30 \mathrm{~W}$ RF power. Owing to the high etching rate of $18 \mathrm{~nm} / \mathrm{min}$ of $\mathrm{SiO}_{x}$ in $\mathrm{KOH}$ compared with $1.2 \mathrm{~nm} / \mathrm{min}$ for $\mathrm{SiN}_{x}$, a minimum of $100 \mathrm{~nm}$ thick $\mathrm{SiO}_{x}$ and $20 \mathrm{~nm}$ thick $\mathrm{SiN}_{x}$ is required for hard-masking Si during $\mathrm{KOH}$ etching. Thus, a systematic study on laser-induced blistering and catapulting was carried out on $100 \mathrm{~nm}$ to $300 \mathrm{~nm}$ thick $\mathrm{SiO}_{x}$ and $20 \mathrm{~nm}$ to $266 \mathrm{~nm}$ thick $\mathrm{SiN}_{x}$ film to establish fluence thresholds and exposure windows that produced the smallest ablation craters in hard mask with high reproducibility and minimal collateral damage to expose $\mathrm{Si}$ for alkaline etching. The femtosecond fiber laser (FCPA $\mu$ Jewel D-400-VR, IMRA) output was frequency doubled to $522 \mathrm{~nm}$ via second harmonic generation, and applied at $100 \mathrm{kHz}$ pulse repetition rate to avoid cumulative heating effects by such rapidly arriving laser pulses. The beam was linearly polarized and of 170 fs pulse duration. By monitoring the back reflection on a CCD camera, a planoconvex lens of $8 \mathrm{~mm}$ focal length (5724-H-A New Focus) focused the Gaussian-shaped laser beam to a diffractionlimited spot size of $1.25 \mu \mathrm{m}$ diameter $\left(1 / \mathrm{e}^{2}\right)$ precisely onto the sample surface that was mounted on a XY motorized stages (ABL1000, Aerotech). A linear polarizer and waveplate power attenuator varied the laser pulse energy between 0.2 and $1.25 \mathrm{~nJ}$ to drive different levels of surface modification in hard masks of various thicknesses. Additional laser processing details are provided in reference [15]. Further, the smallest craters in all the films were tested for effective anisotropic etching in $30 \mathrm{wt} \%$ aqueous solution of $\mathrm{KOH}$ maintained at $60{ }^{\circ} \mathrm{C}$ using a two-step procedure. First, the sample was etched for $30 \mathrm{~s}$, and then cleaned and washed in DI water followed by nitrogen drying. Second, the sample was etched again over a variable time to yield a high-fidelity inverted-pyramid structure. The first step served to mainly remove ablation debris.

The fluence threshold for blistering and catapulting of $\mathrm{SiN}_{x}$ was found to increase strongly from $0.29 \mathrm{~J} / \mathrm{cm}^{2}$ and $0.45 \mathrm{~J} / \mathrm{cm}^{2}$, respectively, for a $20 \mathrm{~nm}$ thick film to $0.67 \mathrm{~J} / \mathrm{cm}^{2}$ and $1.02 \mathrm{~J} / \mathrm{cm}^{2}$, respectively for a $266 \mathrm{~nm}$ thick film. However, the values modulate with varying FabryPérot interference effects as the optical film thickens. Over this film thickness range, the minimum ablation crater diameter was found to increase monotonically from $0.6 \mu \mathrm{m}$ to $\sim 2 \mu \mathrm{m}$. Generally, higher fluence was necessary to generate larger internal ablation pressure to delaminate and lift thicker films, but at the cost of creating larger diameter craters in the c-Si substrate. The observed fluence thresholds and the ablation crater diameters were nearly identical in $\mathrm{SiO}_{x}$ films in comparison with $\mathrm{SiN}_{x}$ films of the same thickness, and are in accord with the results reported for $147 \mathrm{~nm} \mathrm{SiO}{ }_{x}$ films [13]. During KOH etching, the smallest ablation craters in $\geqslant 100 \mathrm{~nm}$ thick $\mathrm{SiN}_{x} / \mathrm{SiO}_{x}$ films did not etch to form inverted-pyramids. This is attributed to the high fluence $\left(\geqslant 0.96 \mathrm{~J} / \mathrm{cm}^{2}\right)$ used to delaminate $\geqslant 100 \mathrm{~nm}$ thick films that crystallographically damages the underlying $\mathrm{Si}$ and thus rendering it unetchable in $\mathrm{KOH}$ [16]. The results show that the thinnest possible dielectric film is favoured to yield the smallest ablation crater diameter with minimum Si damage. Hence, a $20 \mathrm{~nm} \mathrm{SiN}_{x}$ film was deemed optimal for creating the smallest possible mask aperture with sufficient thickness to resist $\mathrm{KOH}$ etching.

The blister dynamics observed in $20 \mathrm{~nm} \mathrm{SiN}_{x}$ with increasing laser fluence was similar to that reported for a $100 \mathrm{~nm} \mathrm{SiO}_{x}$ film [17]. The blister grows in diameter until a threshold for the perforation of the blister is reached at which point a nano-hole is formed, followed by mechanical ejection of the blistered $\mathrm{SiN}_{x}$ film at higher fluences, leaving behind approximately a $50 \mathrm{~nm}$ deep crater in the underlying Si substrate. Figure 2a shows scanning electron micrographs (SEMs) of this sequence beginning with the threshold for blister formation $\left(0.29 \mathrm{~J} / \mathrm{cm}^{2}\right)$, and following with the threshold for perforating the blister with a nanohole $\left(0.31 \mathrm{~J} / \mathrm{cm}^{2}\right)$, the collapse of a blister with a nanohole $\left(0.41 \mathrm{~J} / \mathrm{cm}^{2}\right)$, the catapulting threshold fluence for mechanically ejecting the blister $\left(0.45 \mathrm{~J} / \mathrm{cm}^{2}\right)$, and the catapulted blister at a fluence above the threshold $\left(0.49 \mathrm{~J} / \mathrm{cm}^{2}\right)$. The corresponding atomic force micrographs and line profiles of this morphology are shown in Figures $2 \mathrm{~b}$ and 2c, respectively, while Figure 2d shows SEMs of the corresponding features after $\mathrm{KOH}$ etching for $2.5 \mathrm{~min}$ (step1: $30 \mathrm{~s}$, step2: $2 \mathrm{~min}$ ). The blistered $\mathrm{SiN}_{x}$ layer (intact, perforated and collapsed) was found to protect the underlying Si from $\mathrm{KOH}$ etching whereas the unprotected $\mathrm{Si}$ crater formed due to a blister catapulting $\left(\geqslant 0.45 \mathrm{~J} / \mathrm{cm}^{2}\right)$ resulted in typical inverted-pyramid structure after $\mathrm{KOH}$ treatment. Further, the ablation crater diameter and the effect of $\mathrm{KOH}$ etching was, comprehensively, examined over a broad range 
(a)

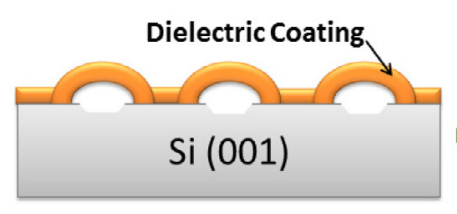

Blistering (b)

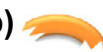

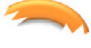

Ablation Crater

$\Rightarrow$

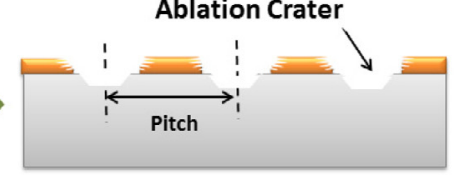

Pattern of Catapulted Blisters (c)

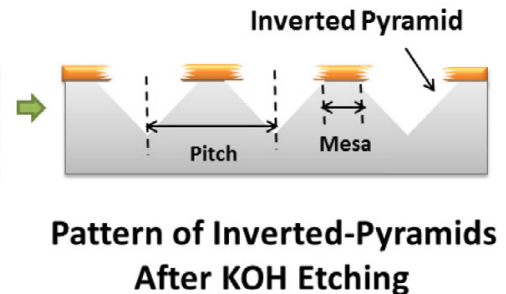

Fig. 1. A schematic illustrating (a) blistering of a dielectric coating upon interaction with femtosecond pulses, (b) catapulting of the blisters at higher laser fluence to form a pattern of shallow ablation craters in c-Si, and (c) the resulting inverted-pyramid structure following $\mathrm{KOH}$ etching.

of laser exposure conditions to determine the smallest possible inverted-pyramid structure that could be reproducibly formed with the tightest packing density and the results are summarized in Figure 2e. The graph shows the crater diameter and the resulting inverted-pyramid width after etching in $\mathrm{KOH}$ for 2.5 min to increase from $0.6 \mu \mathrm{m}$ and $1.13 \mu \mathrm{m}$, respectively, at $0.43 \mathrm{~J} / \mathrm{cm}^{2}$ to $1.25 \mu \mathrm{m}$ and $1.31 \mu \mathrm{m}$, respectively, at $0.53 \mathrm{~J} / \mathrm{cm}^{2}$ fluence. The degree of undercutting increases dramatically as the fluence decreases towards the catapulting threshold, below which only blistering is observed. This is attributed to the decrease in laser-induced Si damage at low fluence. The undercutting was essential to etch beyond the laser damage zone in the c-Si surface to expose damage free (111) planes. The increasing damage apparent in the SEM images (Fig. 2e inset) with increasing laser fluence required longer etching times to compensate for damage but with the trade-off of forming larger sized inverted-pyramid structures. The smallest $(1.13 \mu \mathrm{m})$ clean inverted-pyramid was reproducibly formed at $0.45 \mathrm{~J} / \mathrm{cm}^{2}$ whereas a $1.07 \mu \mathrm{m}$ wide inverted-pyramid was formed at $0.43 \mathrm{~J} / \mathrm{cm}^{2}$ with only $33 \%$ reproducibility.

A high density grid of inverted-pyramids was next investigated using the optimized $0.45 \mathrm{~J} / \mathrm{cm}^{2}$ fluence exposure. At $100 \mathrm{kHz}$ repetition rate, the pitch $(\Lambda)$ of craters along the scanning direction $(x)$ was examined over a range of $\Lambda_{x}=1.2$ to $1.5 \mu \mathrm{m}$ by varying the scan speed between 120 to $150 \mathrm{~mm} / \mathrm{s}$, while line-to-line offsets (y-direction) of $\Lambda_{y}=1.2$ to $2 \mu \mathrm{m}$ were tested to create tightly packed arrays with minimum collateral damage. The densest packing was found for $1.5 \mu \mathrm{m}$ spacing in both directions, yielding the grid of craters shown in Figure 3a that etched into a high-fidelity array of invertedpyramids seen in top and cross-sectional views (inset) in Figure $3 \mathrm{~b}$. In the array of ablation craters produced at optimized fluence, the inverted-pyramid size can be varied from $1.13 \mu \mathrm{m}$ to $1.3 \mu \mathrm{m}$ by increasing the $\mathrm{KOH}$ etching time from 2.5 to $5 \mathrm{~min}$, leaving a $370 \mathrm{~nm}$ to $200 \mathrm{~nm}$ wide flat mesa, respectively, between the inverted-pyramids. A further increase in the inverted-pyramid size would lead to occasional over-etching that manifests in the fusion of neighboring pyramids. Larger area SEM observation of the inverted-pyramidal texture (Fig. 3c) did not reveal any pyramid defects over our whole sample set $(\sim 14,000$ holes viewed) in spite of a non-cleanroom processing environment, suggesting a high reproducibility of the devised technique with less than 1 defect per $10^{4}$ holes.

\section{Flexibility offered by the texturing process}

The method offers the advantage of independently varying pattern pitches in $x$ - and $y$-directions with the help of computer-controlled motion stages, as illustrated in Figure 3d. Also, the laser exposure can be modulated during wafer scanning with the help of an acoustic optical modulator (AOM) driven by position-synchronized output (PSO) of the motion stages to create patterns of inverted-pyramids; this is illustrated through the creation of the University of Toronto crest which is shown in SEM and optical images (Figs. 3e and 3f, respectively). A micro-pattern of the inverted-pyramid structure is shown in Figure 3e (inset). In the optical image, the bare silicon appears bright, whereas the texturized regions appear dark, demonstrating their strong optical anti-reflection property.

\section{Optical performance}

After optimization, $2 \mathrm{~cm} \times 2 \mathrm{~cm}$ textures with inverted pyramids of $1.13 \mu \mathrm{m}$ and $1.3 \mu \mathrm{m}$ sizes arrayed at our highest pitch density $\left(\Lambda_{x}=\Lambda_{y}=1.5 \mu \mathrm{m}\right)$ were fabricated to measure the anti-reflection efficacy and the effect of mesa width on the anti-reflectance efficacy of the textures. Figures $4 \mathrm{a}$ and $4 \mathrm{~b}$ show total reflectance at normal incidence and specular reflectance at different angles of incidence, respectively, together with the total reflectance spectrum measured for a bare Si wafer. With $1.13 \mu \mathrm{m}$ wide invertedpyramids, the surface reflectance was reduced to an average of $18.6 \%$ compared with untextured silicon which reflects $34.7 \%$ of incident light in the wavelength range $400 \mathrm{~nm}-1000 \mathrm{~nm}$. For $1.3 \mu \mathrm{m}$ pyramids, the reflectance was reduced to $13.6 \%$ due to the $42 \%$ decrease in the width of mesas. For the same sample, the total reflectance was further reduced to $4 \%$ with the addition of an incompletely optimized $70 \mathrm{~nm}$ thick $\mathrm{SiN}_{x}$ anti-reflective coating (ARC). Calculated optical reflectivity curves are also plotted for the cases of $1.3 \mu \mathrm{m}$ inverted-pyramids (with $200 \mathrm{~nm}$ 

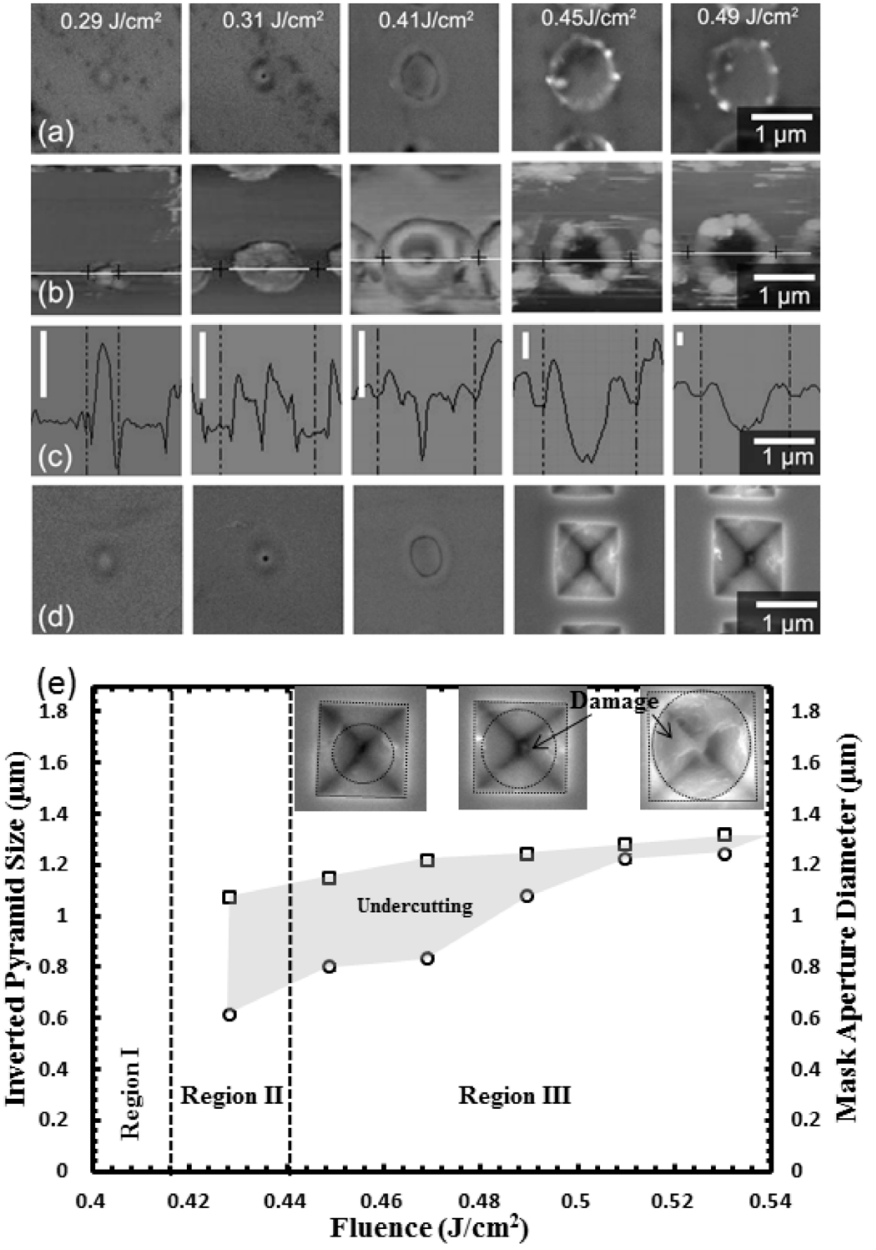

Fig. 2. (a) SEM images of surface modifications in a $20 \mathrm{~nm}$ $\mathrm{SiN}_{x}$ film on c-Si as a function of laser fluence. Corresponding atomic force micrographs and line profiles of the features are shown in (b) and (c), respectively. The vertical scale bar is $20 \mathrm{~nm}$ in each panel of (c). (d) Shows post-etching SEM micrographs of the morphology shown in (a), when etched in $\mathrm{KOH}$ for $2.5 \mathrm{~min}$. The ablation crater diameter (circular marker (o)) and the inverted-pyramid size (square marker $(\square)$ ) observed after 2.5 min $\mathrm{KOH}$ etching are shown in (e) together with select SEM images as a function of fluence. The mask aperture and the inverted pyramid are outlined by a dashed circle and a square, respectively, in each of the SEM images. Fluence zones for blistering (Region I) and low (Region II) and high (Region III) reproducible ejection are identified.

mesas) and the untextured Si surface. Optical wave analysis based on the scattering matrix method [18] was used to simulate the total optical reflectance at normal incidence over the $280 \mathrm{~nm}-1000 \mathrm{~nm}$ spectrum. Reflectivity of the inverted pyramid texture on a $400 \mu \mathrm{m}$ thick polished silicon wafer was calculated for a set pitch of $1.5 \mu \mathrm{m}$ and inverted pyramid size of $1.13 \mu \mathrm{m}$ and $1.3 \mu \mathrm{m}$. For $70 \mathrm{~nm}$ thick $\mathrm{SiN}_{x}$ PECVD antireflective coating, the optical constants were experimentally obtained by ellipsometry. The simulated and measured curves match in the case of untextured $\mathrm{Si}$, whereas a slight incongruity is seen for the $1.3 \mu \mathrm{m}$ inverted-pyramid texture; this deviation
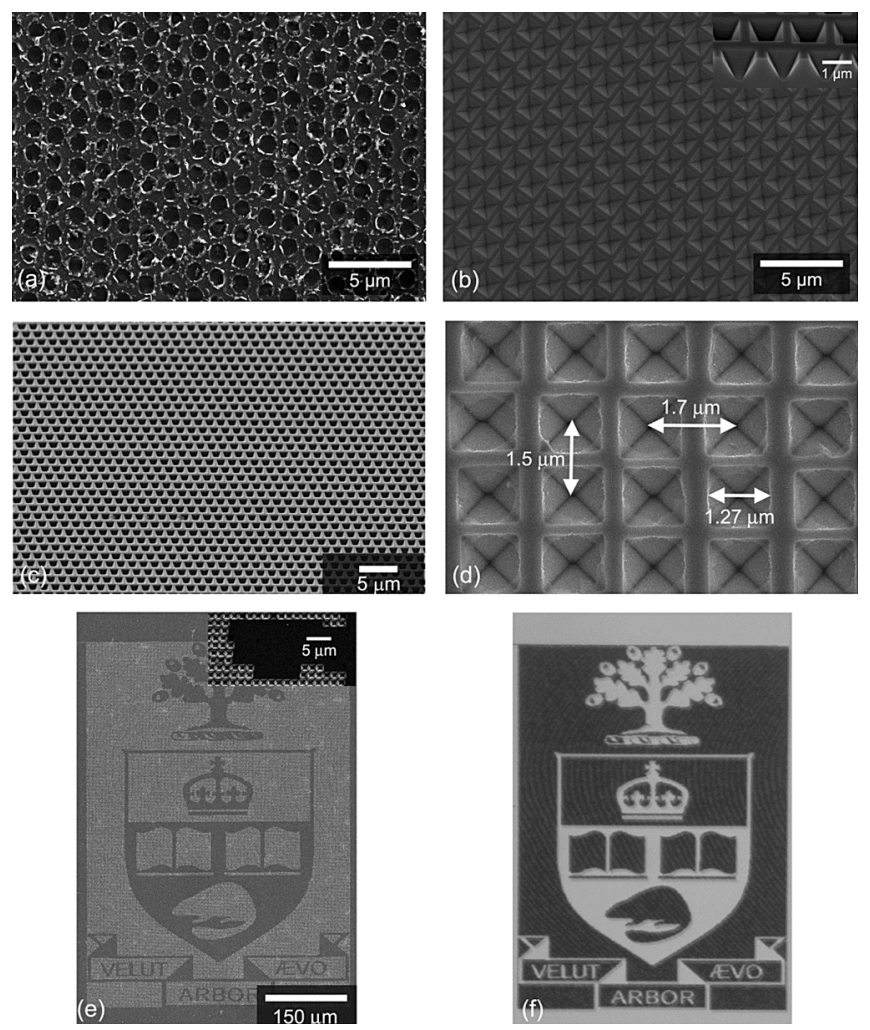

Fig. 3. (a) Top view of a Si wafer with a grid of catapulted blisters at $1.5 \mu \mathrm{m}$ spacing. $\mathrm{KOH}$ etching results in a clean array of $1.3 \mu \mathrm{m}$ inverted-pyramids seen in top and cross-sectional views (inset) (b) and in a large area oblique-view (c). The defect-free texture indicates the high reproducibility offered by the devised technique. (d) Shows an array of $1.27 \mu \mathrm{m}$ sized invertedpyramids placed at a $\Lambda_{x}=1.5 \mu \mathrm{m}$ and $\Lambda_{y}=1.7 \mu \mathrm{m}$. (e) SEM image of a University of Toronto crest micro-patterned with inverted-pyramid structure shown in (e) (inset) demonstrates the capability of the technique to selectively texture areas, leaving untextured planar areas for front contacts. The patterned areas appear bright in this image. (f) The optical image of the crest demonstrates the strong anti-reflective effect in the textured areas.

is possibly due to our theoretical assumption of a square grid arrangement of inverted pyramids, whereas the laboratory sample had skewed alignment of adjacent rows due to the limited control available in the motion stages. The average specular reflectance over $280 \mathrm{~nm}-1000 \mathrm{~nm}$ for the AR coated (70 nm thick $\mathrm{SiN}_{x}$ deposited by PECVD) texture with $1.13 \mu \mathrm{m}$ inverted-pyramids lies below $2 \%$ for incidence angles of $8^{\circ}$ to $40^{\circ}$ and rapidly increases to $3 \%$, $4.6 \%$ and $7.9 \%$ for incident angles of $48^{\circ}, 56^{\circ}$ and $64^{\circ}$, respectively, indicating better optical performance when compared with the grid-less PERL-cell [19] that is also plotted in Figure 4b for comparison.

\section{Efficiency calculations}

Given the strong anti-reflective characteristics observed in the present inverted-pyramid structures, it is instructive to simulate the cell efficiency as a function of 
K. Kumar et al.: Ultrafast laser direct hard-mask writing for high efficiency c-Si texture designs

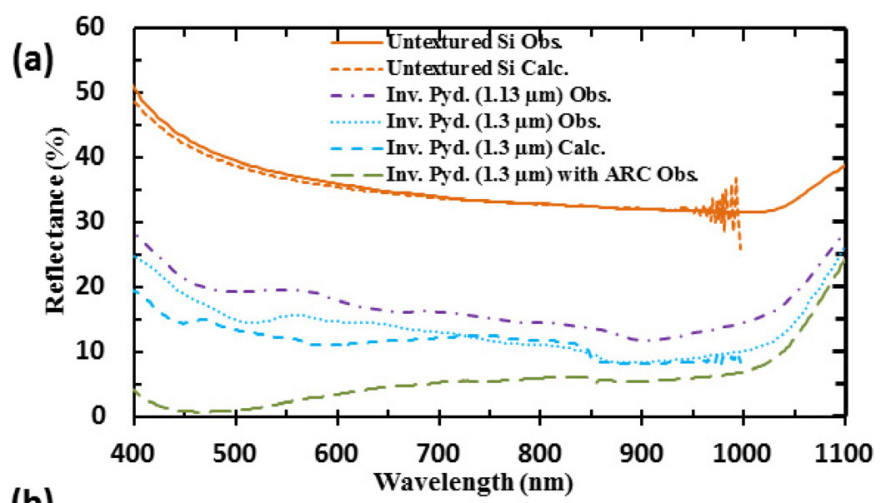

(b)

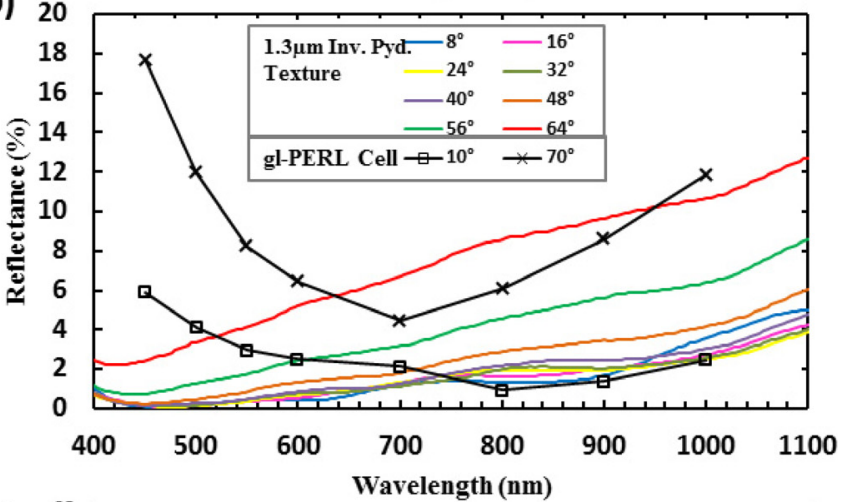

(c)

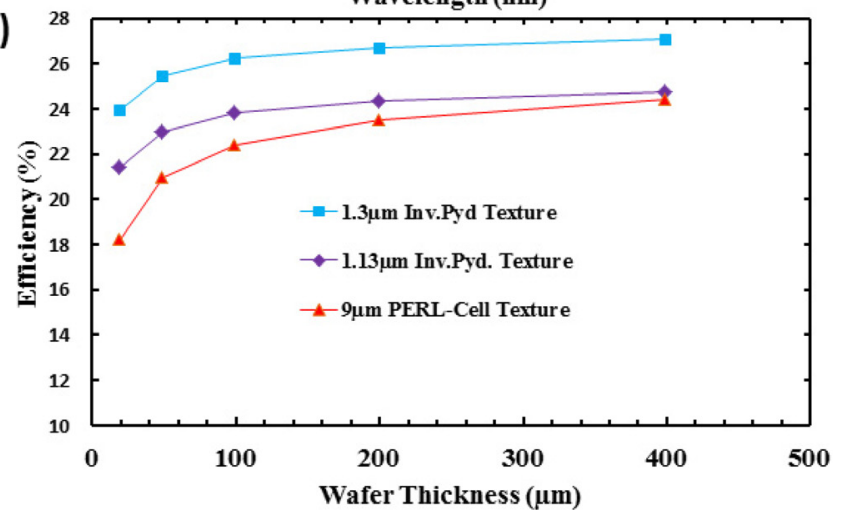

Fig. 4. (a) Experimental (solid lines) and simulated (dashed lines) total reflectance spectra at normal incidence from fabricated samples $(1.13 \mu \mathrm{m}$ and $1.3 \mu \mathrm{m}$ inverted-pyramids) and bare silicon as a function of wavelength. (b) Measured specular reflectance spectra from $1.3 \mu \mathrm{m}$ inverted pyramid texture coated with a $70 \mathrm{~nm}$ thick $\operatorname{SiN}_{x} \mathrm{ARC}$ and from a grid-less PERL-cell with dual layer ARC [19] for the various incidence angles shown. (c) Predicted efficiency of a solar cell with the presently fabricated (1.13 and $1.3 \mu \mathrm{m}$ inverted-pyramid) and the PERL-cell textures at various wafer thicknesses. A $70 \mathrm{~nm}$ thick PECVD deposited $\mathrm{SiN}_{x}$ ARC is considered in efficiency calculations.

wafer thickness for the textures fabricated in this study and the $9 \mu \mathrm{m}$ inverted-pyramidal texture with $\Lambda_{x}=\Lambda_{y}=$ $10 \mu \mathrm{m}$ that was used in the grid-less PERL-cell. Inverted pyramids were placed on the light facing side of the wafer, etched in to a polished silicon wafer of a given thickness. A $70 \mathrm{~nm}$ thick $\mathrm{SiN}_{x}$ ARC and a perfect back reflector were assumed for the front and rear surfaces, respectively, in both cases. For efficiency calculations, the total number of photons absorbed in the solar cell $\mathrm{N}_{p h}$ was calculated by integrating absorption of AM1.5 solar radiation over the wavelength range of $280 \mathrm{~nm}-1000 \mathrm{~nm}$. Consistent with Feng et al. [20], a collection efficiency of $85 \%$ was assumed. The short circuit current was given by $J_{s c}=0.85 \times e N_{p h}$, where $e$ is the electronic charge. The open circuit voltage was obtained from $V_{o c}=(k T / e) \ln \left(J_{s c} / J_{o}+1\right)$, where $\mathrm{k}$ is the Boltzmann constant, $T=300 \mathrm{~K}$, and $J_{o}$ is the reverse bias saturation current. Given that the smallest $J_{o}$ value of about $10^{-15} \mathrm{~A} \mathrm{~cm}^{-2}[21]$ for Si at $300 \mathrm{~K}$, here we use $J_{o}=1.5 \times 10^{-15} \mathrm{~A} \mathrm{~cm}^{-2}$. A fill factor of $80 \%$ was assumed, representing an achievable value for a well-designed photovoltaic device operating at the maximum power point [21]. Finally, the solar cell efficiency was expressed as $\eta=0.8 \times J_{s c} \times V_{o c} / P_{\mathrm{AM} 1.5}$, where $P_{\mathrm{AM} 1.5}=0.1 \mathrm{~W} \mathrm{~cm}^{-2}$ is the incident power of AM1.5 solar radiation.

The simulation results (Fig. 4c) show a moderate efficiency gain of $2.7 \%$ and $0.4 \%$ for the $1.3 \mu \mathrm{m}$ and $1.13 \mu \mathrm{m}$ inverted-pyramids, respectively, in relation to the $24.4 \%$ for the PERL-cell texture in the case of a $400 \mu \mathrm{m}$ thick wafer. Smaller mesas are clearly favoured as expected, while the smaller pyramids on the size scale of optical wavelengths benefit from reduced reflection due to the graded refraction index effect. However, much stronger enhancement is found in thinner wafers. Efficiency gain of $5.7 \%$ is obtained in the case of $1.3 \mu \mathrm{m}$ sized inverted pyramids on $20 \mu \mathrm{m}$ thin wafers in relation to the $18.2 \%$ PERL-cell texture, due to the enhanced light trapping from the larger diffraction angles possible in the smaller periodic surface structure that directs the weakly absorbing infrared light laterally and thus resulting in a marked increase in performance in such thin wafers. Further, the $1.3 \mu \mathrm{m}$ texture effectively etches less silicon for a given wafer thickness compared to the PERL-cell texture, and hence it provides more silicon for light absorption.

\section{Results and discussion}

We have demonstrated a simple and versatile hardmask writing technique for inverted-pyramid texturing of c-Si with texture feature size and pitch on the order of wavelength. The technique uses individual laser pulses to define the pattern of catapulting blisters in a $20 \mathrm{~nm}$-thin $\mathrm{SiN}_{x}$ layer to expose the underlying c-Si with minimal laser damage, which is then effectively etched with $\mathrm{KOH}$ to form a high fidelity inverted-pyramidal texture. The technique offers control over the periodicity through the use of computer controlled motion stages whereas feature size can be modulated by varying laser fluence or alternatively by chemical etching time. It also offers precise control of the patterned areas. Further, the proposed patterning technique along with the feature size of the order of wavelength leads to minimal removal of silicon $(\sim 1 \mu \mathrm{m})$ and consequently has beneficial implications for high-efficiency ultra-thin $(\sim 20 \mu \mathrm{m})$ silicon PV where excess Si for etching 
is not available. A relative Si material savings of $\sim 80 \%$ is anticipated when using our inverted pyramids of $1.3 \mu \mathrm{m}$ size in comparison with the $9 \mu \mathrm{m}$ PERL-cell inverted pyramids.

Detailed optical wave calculations on a $400 \mu \mathrm{m}$ thick wafer suggest relative efficiency enhancement of $11 \%$ and $1.4 \%$ for the $1.3 \mu \mathrm{m}$ and $1.13 \mu \mathrm{m}$ inverted-pyramids, respectively, when compared with the PERL-cell texture of $9 \mu \mathrm{m}$ inverted-pyramids. Light trapping is not imperative in thick wafers and hence, the predicted enhancement in $400 \mu \mathrm{m}$ thick wafer is mainly due to the graded refractive index effectively formed by the inverted-pyramids of the order of wavelength that couples more light into the Si substrate thereby increasing the cell efficiency. Smaller mesas result in stronger enhancement as expected. However, in thin wafers the amount of light trapping significantly affects the cell efficiency. Specifically, for a $20 \mu \mathrm{m}$ thick wafer a much stronger enhancement of $31.6 \%$ and $17.7 \%$ is calculated in the case of $1.3 \mu \mathrm{m}$ and $1.13 \mu \mathrm{m}$ inverted-pyramids, respectively, relative to the PERL-cell texture. This enhancement in efficiency is due to two factors. First, due to the diffraction of light from the fabricated textures that directs the weakly absorbing infrared light laterally which is effectively absorbed in such thin wafers. Second, $1.3 \mu \mathrm{m}$ inverted pyramid texture etches $\sim 80 \%$ less Si and hence offers more material for light absorption relative to the PERL-cell texture.

In the present study, catapulted blisters were formed at the rate of $10^{5} \mathrm{~s}^{-1}$ with $0.55 \mathrm{~mW}$ laser power. The current development of $\geqslant 100 \mathrm{~W}$ femtosecond lasers together with multi-lens focusing arrays suggests a $\sim 2 \times 10^{5}$ fold increase in the processing rate, i.e., $2 \times 10^{10}$ blisters per second, equivalent to $200 \mathrm{~cm}^{2}$ area production per second that can meet the requirements of current c-Si solar cell manufacturing processes. With further advances in lasers and beam delivery methods the proposed approach has the potential of becoming a practical texturing technique for high-efficiency thin c-Si photovoltaics.

This work was supported by the Natural Sciences and Engineering Research Council of Canada, the Ontario Research Fund - Research Excellence program, and the University of Toronto.

\section{References}

1. K.A. Munzer, K.T. Holdermann, R.E. Schlosser, S. Sterk, IEEE Trans. Electron Devices 46, 2055 (1999)

2. C.T.M. Group, International Technology Roadmap for Photovoltaics (ITRPEV.net) Results 2010 2, (2011)

3. D. Munoz, P. Carreras, J. Escarre, D. Ibarz, S. Martin de Nicolas, C. Voz, J.M. Asensi, J. Bertomeu, Thin Solid Films 517, 3578 (2009)

4. H. Sai, Y. Kanamori, K. Arafune, Y. Ohshita, M. Yamaguchi, Prog. Photovolt. Res. Appl. 15, 415 (2007)

5. C.H. Sun, W.-L. Min, N.C. Linn, P. Jiang, B. Jiang, J. Vac. Sci. Technol. B 27, 1043 (2009)

6. C.M. Hsu, S.T. Connor, M.X. Tang, Y. Cui, Appl. Phys. Lett. 93, 133109 (2008)

7. C.H. Sun, W.L. Min, N.C. Linn, P. Jiang, B. Jiang, Appl. Phys. Lett. 91, 231105 (2007)

8. G. Kumaravelu, M.M. Alkaisi, A. Bittar, in Proc. of the 29th IEEE Photovoltaic Specialists Conference, New Orleans, 2002, pp. 258-261

9. Y. Kanamori, M. Sasaki, K. Hane, Opt. Lett. 24, 1422 (1999)

10. M. Moynihan, CircuiTree 22, 16 (2009)

11. J. Zhao, A. Wang, Martin A. Green, F. Ferrazza, Appl. Phys. Lett. 73, 1991 (1998)

12. M.A. Green, K. Emery, Y. Hishikawa, W. Warta, E.D. Dunlop, Prog. Photovolt. Res. Appl. 19, 565 (2011)

13. B.R. Tull, J.E. Carey, E. Mazur, J.P. McDonald, S.M. Yalisove, MRS Bull. 31, 626 (2006)

14. J.P. McDonald, A.A. McClelland, Y.N. Picard, S.M. Yalisove, Appl. Phys. Lett. 86, 264103 (2005)

15. K. Kumar, K.K.C. Lee, P.R. Herman, J. Nogami, N.P. Kherani, Appl. Phys. Lett. 101, 222106 (2012)

16. T. Rublack, M. Schade, M. Muchow, H.S. Leipner, G. Seifert, J. Appl. Phys. 112, 023521 (2012)

17. T. Rublack, S. Hartnauer, P. Kappe, C. Swiatkowski, G. Seifert, Appl. Phys. A 103, 43 (2011)

18. A. Chutinan, N.P. Kherani, S. Zukotynski, Opt. Express 17, 8871 (2009)

19. A. Parretta, A. Sarno, P. Tortora, H. Yakubu, P. Maddalena, J. Zhao, A. Wang, Opt. Commun. 172, 139 (1999)

20. N.-N. Feng, J. Michel, J. Michel, L. Zeng, J. Liu, C.-Y. Hong, L.C. Kimerling, X. Duan, IEEE Trans. Electron Devices 54, 1926 (2007)

21. S.M. Sze, Physics of semiconductor devices, 2nd edn. (Wiley, New York, 1981)

Cite this article as: Kitty Kumar, Kenneth K.C. Lee, Jun Nogami, Peter R. Herman, Nazir P. Kherani, Ultrafast laser direct hard-mask writing for high efficiency c-Si texture designs, EPJ Photovoltaics 4, 45101 (2013). 